\begin{tabular}{|l|l|l||}
\hline \multicolumn{2}{|c|}{ PublisherInfo } \\
\hline \hline PublisherName & $:$ & BioMed Central \\
\hline \hline PublisherLocation & $:$ & London \\
\hline \hline PublisherImprintName & $:$ & BioMed Central \\
\hline \hline
\end{tabular}

\title{
Peptide inhibition of a cytokine response
}

\begin{tabular}{|l|l|l||}
\hline \multicolumn{2}{|c|}{ ArticleInfo } \\
\hline \hline ArticleID & $:$ & 173 \\
\hline \hline ArticleDOI & $:$ & $10.1186 /$ ar-2000-66823 \\
\hline \hline ArticleCitationID & $:$ & 66823 \\
\hline \hline ArticleSequenceNumber & $:$ & 130 \\
\hline \hline ArticleCategory & $:$ & Paper Report \\
\hline \hline ArticleFirstPage & $:$ & 1 \\
\hline \hline ArticleLastPage & $:$ & 3 \\
\hline \hline & & RegistrationDate : 2000-6-29 \\
\hline ArticleHistory & $:$ & OnlineDate \\
\hline \hline ArticleCopyright & $:$ & Current Science Ltd2000-6-29 \\
\hline \hline ArticleGrants & $:$ & \\
\hline \hline ArticleContext & $:$ & 130753311 \\
\hline \hline
\end{tabular}


Cheryl Smythe, Affl

Aff1 Imperial College School of Medicine, London

\section{Keywords}

Endotoxic shock, IFN-?, NF?B, nuclear import, TNF-a

\section{Context}

Activation of genes encoding tumour necrosis factor (TNF)-a, interferon (IFN)-? or interleukin (IL)-1 in response to pro-inflammatory agonists involves the nuclear translocation of SRTFs such as nuclear factor kappa B (NF?B) or nuclear factor of activated T cells (NFAT), and their subsequent interaction with the promoter region of the specific gene. Disruption of the SRTF signalling pathway would suppress the expression of TNF-a and IFN-?, cytokines that are known to play a key role in the systemic inflammatory response syndrome induced by LPS. A linear peptide (SN50) containing a membrane translocation motif and a nuclear localisation sequence (NLS), derived from the p50-NF?B1 subunit of the transcription factor NF?B, was therefore designed and shown to inhibit the nuclear import of NF?B in human monocytic and murine endothelial cells stimulated with LPS or TNF-a. SN50 also inhibited the expression of downstream SRTF-regulated genes such as cycloxygenase and IL-2 in vitro and was shown to interact with a cytoplasmic NLS receptor comprised of the Rch 1/importin (karyopherin)-? heterodimer. To inhibit SRTF mediated inflammatory responses in vivo using a cyclic peptide.

\section{Significant findings}

Fluorescence confocal laser scanning microscopy demonstrated intracellular accumulation of the FITC-cSN50 peptide in RAW 264.7 macrophages. The cyclic peptide was 3-10 times more effective than its linear analogue at inhibiting nuclear import of NF?B in LPS-stimulated cells. Nuclear import of activating protein (AP)-1 and NFAT in stimulated Jurkat T cells was also inhibited by the cSN50 peptide. FACS analysis of blood and spleen cells from mice intraperitoneally injected with FITC-cSN50 demonstrated the rapid delivery of the cyclic peptide to these cells. Injection of LPS into animals has previously been shown to lead to an early rise in pro-inflammatory mediators of endotoxic shock such as TNF-\&\#945, IFN-? and IL-1. In order to test whether cSN50 would inhibit cytokine gene expression in vivo, peptide-treated and saline-treated control mice were injected with LPS and plasma levels of cytokines were measured. Increases in both TNF-a and IFN-? following LPS treatment were significantly suppressed by the cSN50 peptide. Injection of cSN50 peptide also significantly increased the survival rate in LPS treated mice. LPS control mice and those injected with a mutated control 
peptide died within $72 \mathrm{~h}$ of injection, whereas mice treated with $1.5 \mathrm{mg}$ of the cSN50 peptide, in seven injections taking place from 30 min prior to LPS administration to $12 \mathrm{~h}$ after, demonstrated a $90 \%$ survival rate over $72 \mathrm{~h}$. Injection of the peptide $30 \mathrm{~min}$ after LPS exposure reduced the survival rate to $60 \%$.

\section{Comments}

A previous publication by this group detailed the design of a linear peptide that inhibited nuclear import of a range of stress responsive transcription factors (SRTFs) in vitro. This study furthers this work and examines a cyclic peptide of greater potency than its linear analogue and demonstrates its effectiveness in protecting against lipopolysaccharide (LPS)-induced endotoxic shock in vivo.

Furthermore, the peptide appears to attenuate established endotoxic shock if administered shortly after exposure to LPS. Given the broad specificity of the cyclic peptide, future studies that investigate its efficacy in other models of inflammatory disease will be of interest. Although levels of toxicity observed in animal models may not accurately reflect those subsequently observed in humans, it is important that the mice did not develop any adverse side effects upon treatment with the cyclic peptide. This study identifies a novel suppressor of inflammatory responses, and together with the lack of adverse side effects demonstrated by the treated mice, makes it a potential candidate for therapeutic development.

\section{Methods}

A cell permeable cyclic peptide (cSN50) was generated from the linear SN50 peptide by inserting two cysteine residues to form an intrachain disulphide bond. Peptides were either detected indirectly, using a fluorescently labelled antibody against the epitope tag LMP, or they were first fluorescein isothyanate (FITC) labelled and then detected directly using confocal laser scanning microscopy. In vivo detection of FITC-cSN50 in blood and spleen cells was by fluorescence-activated cell sorting (FACS). Nuclear import of NF?B in RAW 264.7 cells was measured by electrophoretic mobility gel shift assay using a radiolabelled ?B probe. Levels of TNF-a and IFN-? were measured by ELISA. LPS injected female C57BL/6 mice were used to model LPS-induced lethal shock. Histological analysis was carried out by haematoxylin and eosin staining of formalin-fixed, paraffin-embedded sections of the liver, spleen, kidneys, and lungs.

\section{References}

1. Liu XY, Robinson D, Veach RA, Liu D, Timmons S, Collins RD, Hawiger J: Peptide-directed suppression of a pro-inflammatory cytokine response. J Biol Chem. 2000, 275: 16774-16778.

This PDF file was created after publication. 